\title{
WEAK CONSISTENCY IN SAATY'S AHP - EVALUATING CREATIVE WORK OUTCOMES OF CZECH ART COLLEGES
}

\author{
Jan Stoklasa, Věra Jandová, Jana Talašová*
}

\begin{abstract}
The full consistency of Saaty's matrix of preference intensities used in AHP is practically unachievable for a large number of objects being compared. There are many procedures and methods published in the literature that describe how to assess whether Saaty's matrix is "consistent enough". Consistency is in these cases measured for an already defined matrix (i.e. ex-post). In this paper we present a procedure that guarantees that an acceptable level of consistency of expert information concerning preferences will be achieved. The proposed method is based on dividing the process of inputting Saaty's matrix into two steps. First, the ordering of the compared objects with respect to their significance is determined using the pairwise comparison method. Second, the intensities of preferences are defined for the objects numbered in accordance with their ordering (resulting from the first step). In this paper the weak consistency of Saaty's matrix is defined, which is easy to check during the process of inputting the preference intensities. Several propositions concerning the properties of weakly consistent Saaty's matrices are proven in the paper. We show on an example that the weak consistency, which represents a very natural requirement on Saaty's matrix of preference intensities, is not achieved for some matrices, which are considered "consistent enough" according to the criteria published in the literature.

The proposed method of setting Saaty's matrix of preference intensities was used in the model for determining scores for particular categories of artistic production, which is an integral part of the Registry of Artistic Results (RUV) currently being developed in the Czech Republic. The Registry contains data on works of art originating from creative activities of Czech art colleges and faculties. Based on the total scores achieved by these institutions, a part of the state budget subsidy is being allocated among them.
\end{abstract}

Key words: Multiple criteria evaluation, AHP, weak consistency, work of art, Registry of Artistic Results

Received: July 15, 2012

Revised and accepted: November 1, 2012

* Jan Stoklasa, Věra Jandová, Jana Talašová

Department of Mathematical Analysis and Applications of Mathematics, Faculty of Science, Palacky University in Olomouc, E-mail: jan.stoklasa@upol.cz, vera.jandova01@upol.cz, jana.talasova@upol.cz 


\section{Introduction}

When designing mathematical models for such purposes as evaluation of works of art, the evaluators' experience and background needs to be taken into account. Suitable mathematical tools have to be chosen so that the resulting model is not only mathematically sound, but also possible to implement in real setting. Particularly when dealing with abstract categories and large amounts of pairwise comparisons and experts not closely related to the field of mathematics, it is important to find an appropriate way of inputting the data. During each step we might need to go back and correct some partial inconsistencies, but the result should be a reasonably consistent mathematical representation of experts' knowledge concerning their preferencies on the given set of objects. Tools enabling the evaluators to check the consistency of inputted preferences for pairs of objects (even during the process of data input) and guidelines for such purposes can be the key to success in such application areas. We are going to present here a real-life problem and our solution to it. The task was to develop a mathematical model for the evaluation of works of art, which required cooperation with experts from the field of artistic production.

In the second section of the paper, we start with the description of the Registry of Artistic Results (RUV - from Czech "Registr Uměleckých Výstupü"), its purpose and structure. We also introduce the evaluation criteria and the resulting categories of works of art in this section. Section 3 describes the two-step mathematical model used to obtain scores for each category, and the respective evaluation methodology. As consistency is a great issue when using Saaty's matrices of large dimensions, Section 4 discusses various measures of inconsistency in Saaty's matrix and presents a short overview of the relevant research. We introduce here a new concept of weak consistency and prove several properties of weakly consistent Saaty's matrices. Section 5 presents two methods for determining the scores of categories of works of art by Saaty's matrix of preference intensities - the eigenvector method and the logarithmic least squares method. We discuss here the possibility of seeing the data in Saaty's matrix as repeated measurements of relative information concerning the importances in the set of categories of works of art and hence dealing with it as with compositional data. All the results presented in this paper are then summarized and discussed in Section 6.

\section{Classification of Works of Art}

The Registry of Artistic Results has been developed and is currently being pilot tested in the Czech Republic. It contains information on works of art originating from creative activities of art colleges and faculties (see [14]). The RUV is conceived as an analogy to the register of R\&D outcomes where information on outcomes of research institutions (including universities) has been collected for some years already. In both registers the outcomes are stored under several categories. These categories are assigned scores. The sum of scores of all the outcomes of a given university is considered an indicator of its performance in the area of creative activity. These numerical values can then be used in decisions regarding one part of the total money to be allocated among universities from the state budget. The 
structure of the evaluated categories of works of art used in the Registry of Artistic Results was inspired, to some extent, by the artistic categories in the Slovak Republic (see [12]). However, the mathematical model used to determine scores for each category in Slovakia is quite different.

For the purposes of registration of works of art originating from creative activities of the Czech art colleges and faculties, the whole area of artistic production is divided into seven fields: architecture, design, film, fine arts, literature, music and theatre. Each piece of art, regardless of the field, is categorized according to the following three criteria:

- Relevance or significance of the piece;

- Extent of the piece;

- Institutional and media reception/impact of the piece.

In each criterion, three different levels are distinguished (denoted by capital letters for easier handling):

- The criterion Relevance or significance of the piece:

A - a new piece of art or a performance of crucial significance;

$\mathrm{B}$ - a new piece of art or a performance containing numerous important innovations;

$\mathrm{C}-\mathrm{a}$ new piece of art or a performance pushing forward modern trends.

- The criterion Extent of the piece:

$\mathrm{K}$ - a piece of art or a performance of large extent;

$\mathrm{L}$ - a piece of art or a performance of medium extent;

$\mathrm{M}$ - a piece of art or a performance of limited extent.

- The criterion Institutional and media reception/impact of the piece:

$$
\begin{aligned}
& \mathrm{X} \text { - international reception/impact; } \\
& \mathrm{Y} \text { - national reception/impact; } \\
& \mathrm{Z} \text { - regional reception/impact. }
\end{aligned}
$$

The resulting category for a piece of art is given by a combination of three capital letters - e.g. AKX, BKY, or CLZ. There are 27 categories altogether that are assigned a score. The decision concerning the relevance or significance of the piece (choice of $\mathrm{A}, \mathrm{B}$ or $\mathrm{C}$ ) rests upon expert assessment; the experts have at their disposal general definitions of each category and specific real-life (historical) examples of works of art in each category for all 7 fields of artistic production and these examples assist them in the decision process. (Gathering real-life representatives of all the categories for all the fields of arts was also important to confirm a common understanding of the categories and to ensure that corresponding categories are really comparable in terms of evaluation across all the fields of arts.) As for the extent of the piece (levels K, L, M), all the classes are clearly specified for 
all the fields of art. As for the institutional and media reception/impact, lists of institutions corresponding to categories X, Y, Z are available for all fields.

Our task was to develop a mathematical model to determine the scores for such categories of pieces of art (each described by a triplet of capital letters). We have decided to solve this problem by applying Saaty's AHP method (introduced in $[10])$. We need to realize that there are interactions among the three mentioned criteria. For example the first one (expertly defined Relevance or significance of the piece of art) and the third one (Institutional and media reception/impact of the piece) partly overlap. It was, therefore, not possible to use the approach, where first we would determine the weights of the criteria and scores for their individual levels, and then set the scores of categories as respective weighted averages. We did not choose the ANP method either (see [11] for more details) which is able to solve tasks with mutually dependent criteria. It was because deriving information concerning the links among the criteria has proven to be extremely difficult for the experts in the field of arts. We have decided to compare directly the 27 works of art categories. In the case of such a large number of objects (categories), Saaty suggests (see [8]) to split the problem into several smaller ones and then apply the AHP on these. If we chose to do so, we would have to define relative significances for abstract supercategories. This is a difficult task for the experts from the field of arts. The difficulties resulting from a large dimension of the matrix of preference intensities were considered small compared to the difficulties resulting from the use of other ways of solving the problem. For a large number of mutually compared objects the issue of obtaining a Saaty's matrix that is consistent enough arises. Our solution to this problem will be described and further discussed in the following sections.

\section{Determining Scores for Particular Categories of Artistic Production}

Saaty's method (see $[8,6,9])$ served as a basis for determination of scores for all 27 categories of artistic production. No matter how obvious it was that this mathematical tool is the most appropriate for this task, certain challenges concerning its use were also clearly apparent: 1) difficulty for a team of experts to express preferences with respect to abstract categories; 2) reaching consensus within the group of experts (professional guarantors of particular fields of art); and most importantly 3) difficulty to reach acceptable consistency of Saaty's matrix for such a large number of categories (Section 4 deals with this issue). Admittedly, expressing one's opinion on intensities of preferences with respect to abstract categories is difficult. Experts - professional guarantors of particular artistic fields - were first asked to provide examples of works of art in all categories in their field (see Section 2). Next, professional guarantors of each field of art set their preferences concerning pairs of categories, while using the representatives (examples) as an aid in their decision making. Although it would be possible for each of these experts to express their preferences separately, and only then to derive the collective preferences (from the individual ones), we used a different approach. The collective preferences were set directly at a team meeting of experts. The reason was that 
art-college experts are not used to work with mathematical models and individual inputting of required data could prove difficult for them. Achieving consensus was also intentionally preferred over averaging different opinions.

Great effort was made to find the best way of converting expert preferences concerning the 27 categories of artistic production (represented in each field of art by specific examples) into a mathematical model. Such model is required to be a consistent representation of experts' preferences and to allow calculating the scores of all the categories of works of art. To facilitate the process of inputting required data by the experts, to achieve the necessary consistency of this input and to obtain consensual scores for all the categories of works of art, the following two-step procedure was performed. First, a pairwise comparison method was used to determine the order of importance of the 27 categories (their quasi-ordering). Second, a Saaty's matrix was constructed with categories numbered according to this quasiordering. Such matrix of preference intensities was then used to determine scores for the categories.

\subsection{Matrix of preferences and indifferences}

In the first step, we have determined the order of importance of the categories by the Pairwise Comparison Method (see [6, 13]). This method employs a matrix of preferences and indifferences $P=\left\{p_{i j}\right\}_{i, j=1, \ldots, 27}$. For its elements it holds that:

$p_{i j}=1$, if the $i^{\text {th }}$ category is more important than the $j^{\text {th }}$ category;

$p_{i j}=0.5$, if the $i^{t h}$ category is equally important as the $j^{t h}$ category;

$p_{i j}=0$, if the $j^{\text {th }}$ category is more important than the $i^{\text {th }}$ category.

It is sufficient for the experts to fill in the upper right triangle of the matrix, that is, the elements $p_{i j}, i<j$, as $p_{i i}=0.5$ and $p_{j i}=1-p_{i j}$. The row sums $R_{i}=\sum_{j=1}^{27} p_{i j}, i=1, \ldots, 27$, are used in this method to determine the order of the mutually compared objects according to their significance. To be able to accept the results of this method, we need to be sure that the matrix $P$ defined by experts contains consistent information on their preferences on the set of objects. The matrix $P$, therefore, has to represent a quasi-ordering of objects, i.e. a complete and transitive relation (a relation that can be described as a linear ordering of classes of indifferent objects). The completeness of this relation is ensured by the process of inputting of matrix $P\left(p_{j i}=1-p_{i j}\right)$; the transitivity in the terms of matrix $P$ can be expressed by the following condition:

$$
p_{i k}=\max \left\{p_{i j}, p_{j k}\right\}, \text { for all } p_{i j}, p_{j k} \geq 0.5, i, j, k=1, \ldots, 27 .
$$

If the matrix does not satisfy the condition (1), we make the minimum amount of changes necessary for it to become so. These changes are then consulted with the team of experts and if they are approved of, we can proceed. All the changes actually made while solving our problem are summarized in Fig. 1.

\subsection{Saaty's matrix of preference intensities}

Saaty's matrix of preference intensities for $n$ mutually compared objects is a square matrix $S=\left\{s_{i j}\right\}_{i, j=1}^{n}$, that is reciprocal (i.e. $s_{i j}=1 / s_{j i}$ for all $i, j=1,2, \ldots, n$ ) 


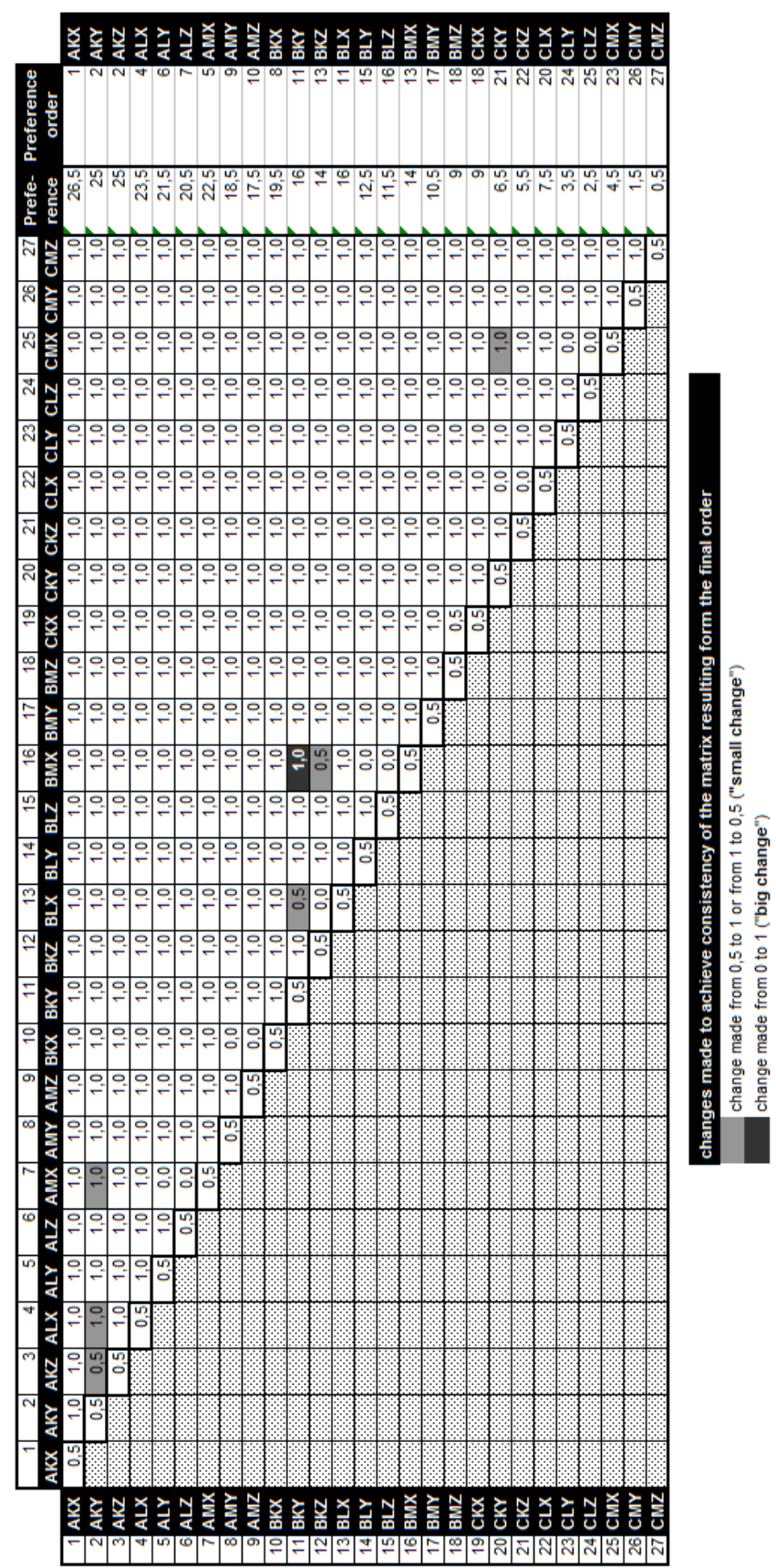

Fig. 1 Pairwise comparison matrix for 27 categories. Necessary changes are highlighted. 
and for an object $i$ that is more or equally preferred than object $j$ the element $s_{i j} \in\{1,2,3,4,5,6,7,8,9\}$. Tab. I provides the linguistic descriptors of these numerical values for Saaty's scale. If, for example, $s_{i j}=3$, it can be interpreted that the object $i$ is 3 times as important as the object $j$. From Perron-Frobenius theorem it follows that Saaty' matrix always has a maximum eigenvalue (spectral radius - see [6]). A fully consistent Saaty's matrix has a single nonzero eigenvalue, which is equal to the order of the matrix.

In the second step of our method, Saaty's matrix of preference intensities $S=$ $\left\{s_{i j}\right\}_{i, j=1}^{27}$ was constructed for categories numbered in ascending order according to their significance determined in the previous step. Again, it was in this case sufficient to fill in the upper right triangle of the matrix $S$, as $S$ is reciprocal. The elements $s_{i j}, i<j$, were set using Saaty's scale presented in Tab. I. Before we could proceed with calculating the scores of the categories, the consistency of the information provided by experts through the matrix $S$ had to be checked. It is well known that the full consistency defined by Saaty:

$$
s_{i k}=s_{i j} \cdot s_{j k}, \text { for all } i, j, k=1,2, \ldots, n,
$$

is basically unachievable even for not too large sets of mutually compared objects. Various authors, including Saaty (see [7]), therefore define for the practical use of Saaty's matrix various criteria to decide, whether a Saaty's matrix that is not fully consistent is at least consistent enough to represent expert knowledge concerning the relative preferences on a set of objects that are being compared. Hence, these authors allow some tolerance in the fulfillment of condition (2). We have approached this problem differently. We have defined directly the notion of weak consistency of Saaty's matrix. This natural condition has the advantage that already during the process of inputting data into Saaty's matrix that is constructed for categories ordered in accordance with their significance, the experts can easily check its fulfillment. The details concerning the issue of consistency of Saaty's matrix are given in the following section.

\begin{tabular}{cl}
\hline$s_{i j}$ & linguistic meaning \\
\hline 1 & $i^{\text {th }}$ object is equally important as $j^{\text {th }}$ object \\
3 & $i^{\text {th }}$ object is slightly/moderately more important than $j^{\text {th }}$ object \\
5 & $i^{\text {th }}$ object is strongly more important than $j^{\text {th }}$ object \\
7 & $i^{\text {th }}$ object is very strongly more important than $j^{\text {th }}$ object \\
9 & $i^{\text {th }}$ object is extremely/absolutely more important than $j^{\text {th }}$ object \\
$2,4,6,8$ & correspond with the respective intermediate linguistic meanings \\
\hline
\end{tabular}

Tab. I Saaty's scale.

\section{Consistency and Weak Consistency of Saaty's Matrix}

In this section, we are going to deal with a general Saaty's matrix $S=\left\{s_{i j}\right\}_{i, j=1}^{n}$, which represents the information concerning preference intensities among $n$ ob- 
jects (in our application categories of works of art) given by experts. In the sense of the previously mentioned definition of Saaty's matrix, this means that $s_{i j} \in\{1 / 9,1 / 8,1 / 7, \ldots, 1 / 2,1,2, \ldots, 8,9\}$ and the matrix is reciprocal, i.e. $s_{i j}=1 / s_{j i}$ for all $i, j=1, \ldots, n$. We also require the matrix to be consistent enough to be able to use Saaty's method to calculate the relative importances of the objects.

The full consistency condition of Saaty's matrix is expressed by (2). Such a full consistency is, however, unachievable in real situations. Consider, for example four arbitrary objects linearly ordered according to their importance. If each of them is just slightly more important than the following one, then in the case of full consistency, the first object would have to be 27 times more important as the fourth one. But we have no value greater than 9 available on Saaty's 9 point scale to express our preferences (Tab. I). Saaty [7], therefore, proposes an inconsistency (Saaty introduced it as consistency index) index $(C I)$ based on the spectral radius $\left(\lambda_{\max }\right)$ of the pairwise comparison matrix $S$ :

$$
C I(S)=\frac{\lambda_{\max }-n}{n-1}
$$

where $n$ is the dimension of the matrix $S$. For any Saaty's matrix it holds that $C I(S) \geq 0$. $C I(S)$ was defined by Saaty to introduce an inconsistency measure for Saaty's matrices that would be independent of the dimension of the matrix. The average $C I(S)$ of randomly generated Saaty's matrices, however, proved to be growing as the dimension of the matrix grows. Saaty, therefore, introduced the inconsistency ratio $C R(S)$ :

$$
C R(S)=\frac{C I(S)}{R I(n)},
$$

where $R I(n)$ is the so-called random inconsistency index that represents the inconsistency of a randomly generated reciprocal pairwise comparison matrix of dimension $n$. $R I(n)$ is calculated as an average of indices $C I(S)$ calculated for a set of randomly generated reciprocal pairwise comparison matrices of dimension $n$. Matrix $S$ for which $C R(S)<0.1$ is then considered consistent enough.

\subsection{Other approaches to determining satisfactory level of consistency of Saaty's matrix}

Various authors have been trying to construct alternative measures of inconsistency of the matrix $S$. Alonso \& Lamata [2] pointed out that the use of randomly generated reciprocal pairwise comparison matrices $S$ of dimension $n$ to determine the $R I(n)$ may result in slightly different indices depending on the number of such matrices used to compute the $R I(n)$. As they try to lower the computational complexity of determining $R I(n)$ for larger matrices, they realize that the growth of an average largest eigenvalue $\bar{\lambda}_{\max }(n)$ is easier to predict than the $R I(n)$ as the dimension $n$ of the matrix $S$ grows. For $\bar{\lambda}_{\max }(n)$, the expression $\bar{\lambda}_{\max }(n)=2.7740 n-4.3513$ obtained by the least square method proves to be very accurate and easy to compute. Using $\bar{\lambda}_{\max }(n)$, they compute the random inconsistency index $R I_{\lambda}(n)=\left(\bar{\lambda}_{\max }-n\right) /(n-1)$. These authors, therefore, propose to 
compute $C R(S)$ using the following formula:

$$
C R(S)=\frac{C I(S)}{R I_{\lambda}(n)}=\frac{\lambda_{\max }-n}{\bar{\lambda}_{\max }-n} .
$$

Analogically to Saaty's approach, for $C R(S)<0.1$ is the matrix $S$ considered consistent enough.

Lamata \& Pelaez [5] propose an inconsistency index $C I^{*}$ for a matrix $S$ of type $n \times n$ using determinants:

$$
C I^{*}(S)= \begin{cases}0 & \text { if } n<3 \\ \operatorname{det}(S) & \text { if } n=3 \\ \frac{1}{N T(S)} \sum_{i=1}^{N T(S)} C I^{*}\left(\sigma_{i}\right) & \text { if } n>3\end{cases}
$$

where $\sigma$ is a submatrix of $S$ of type $3 \times 3$ consisting of the rows and columns $i, j, k \in\{1, \ldots, n\}, i \neq j \neq k$, and $N T(S)$ is the number of such submatrices, i.e.

$$
N T(S)= \begin{cases}0 & \text { if } n<3 \\ \frac{n !}{(n-3) ! 3 !} & \text { otherwise. }\end{cases}
$$

Next they generate 10000 random Saaty's matrices of type $n \times n$. For this data they determine the $p$-value (e.g. 0.05$)$ and for this $p$-value a critical value $C R^{*}$ is calculated. If $C I^{*}(S)>C R^{*}$, then the matrix $S$ is considered inconsistent.

Ji \& Jiang [4] find Saaty's scale to be problematic - this paper deals with the transitivity on the linguistic and numerical parts of the scales most commonly used in AHP and with various types of inconsistency causes inherent to the scales used in AHP. They propose a scale that is transitive both in linguistic and in numerical part. The numerical part of this scale consists of the following set of values: $\{0,+0.5,-0.5,+1,-1,+1.5, \ldots,-3.5,+4,-4\}$. A common Saaty's matrix $S$ can be transformed into a matrix $D$ using this new so-called derived transitive scale in the following way:

$$
d_{i j}= \begin{cases}\left(s_{i j}-1\right) / 2 & \text { if } s_{i j} \geq 1 \\ -\left(\frac{1}{s_{i j}}-1\right) / 2 & \text { if } s_{i j}<1\end{cases}
$$

The matrix $D$ is absolutely consistent, if

$$
d_{i j}=\frac{1}{n} \sum_{k=1}^{n} d_{i k}+\frac{1}{n} \sum_{k=1}^{n} d_{k j}=\frac{1}{n} \sum_{k=1}^{n} d_{i k}-\frac{1}{n} \sum_{k=1}^{n} d_{j k}
$$

i.e. if $d_{i j}=\bar{d}_{i}-\bar{d}_{j}$, where $\bar{d}_{i}=\frac{1}{n} \sum_{k=1}^{n} d_{i k}$ and $\bar{d}_{j}=\frac{1}{n} \sum_{k=1}^{n} d_{j k}$. An average grade deviation per comparison is then determined:

$$
\epsilon=\sqrt{\frac{\sum_{i=1}^{n-1} \sum_{j=i+1}^{n}\left[d_{i j}-\left(\bar{d}_{i}-\bar{d}_{j}\right)\right]^{2}}{n(n-1) / 2}} .
$$

The decision maker sets up an acceptable level of deviation ald, and if $\epsilon<$ ald the matrix is considered consistent. 
All of these approaches to assessing the consistency of the matrix of preference intensities are mathematically sound. These approaches, however, forget about the experts that input the data. It is almost impossible for the experts to check whether they are consistent (or consistent enough) in their preferences during the process of inputting data. Which is a major drawback when these experts are far from the field of mathematics and the dimension of the matrix is large. If we obtain from the experts a matrix of preference intensities that is not consistent enough, the usual advice is to start from the beginning and fill the matrix in again. This approach, however, does not make much sense as it does not guarantee that the new matrix of preference intensities will be better (more consistent) than the previous one. We need to find a way of obtaining a better result. Our solution to this problem is presented in the following subsection.

\subsection{Weak consistency}

Unlike most of the authors that start with full consistency and try to determine an acceptable level of its violation (see $[2,5,4]$ ), we have chosen a different approach. We define directly a weak consistency that is based on the properties that should intuitively hold, and we require these properties to be fully met. This is of great use when we need the experts that are expressing their preference intensities to check the consistency themselves during the process of inputting. If we utilize the linguistic meanings of the elements of Saaty's scale, we can define weak consistency such that for example if an object $A$ is slightly more important than object $B$ and object $B$ is strongly more important than object $C$, we need at least the larger of the preference intensities to hold between $A$ and $C$ (which means that a stronger preference than the larger one of these two is also acceptable). For situations, when two objects are equally important, such as if $A$ is equally as important as $B$ and $B$ is very strongly more important than $C$, it is reasonable to require $A$ to be very strongly more important than $C$ (the preference between the two objects that are not indifferent should hold between $A$ and $C$ ). This understanding of weak consistency is summarized in Definition 1.

Definition 1: Let $S=\left\{s_{i j}\right\}_{i, j=1}^{n}$ be Saaty's matrix of preference intensities. We say that $S$ is weakly consistent, if for all $i, j, k \in\{1,2, \ldots, n\}$ the following holds:

$$
\begin{gathered}
s_{i j}>1 \wedge s_{j k}>1 \quad \Longrightarrow \quad s_{i k} \geq \max \left\{s_{i j}, s_{j k}\right\} \\
\left(s_{i j}=1 \wedge s_{j k} \geq 1\right) \vee\left(s_{i j} \geq 1 \wedge s_{j k}=1\right) \quad \Longrightarrow \quad s_{i k}=\max \left\{s_{i j}, s_{j k}\right\} .
\end{gathered}
$$

The property $s_{i k} \geq \max \left\{s_{i j}, s_{j k}\right\}$ can be found as max-max transitivity in the literature [3].

If we order the objects (alternatives) being compared according to their importance from the most important to the least, we get $s_{i j} \geq 1$ for all $i, j=1,2, \ldots, n$ such that $i<j ; s_{i i}=1$ for all $i=1,2, \ldots, n$. The upper triangle of the matrix $S$ then consists only of numbers from $\{1,2, \ldots, 9\}$. In such case, according to the Definition 1, it is sufficient to check whether conditions (10) and (11) are fulfilled for the elements in the upper triangle of $S$ to assess the weak consistency of $S$. 
It is evident that for the weak consistency condition to hold, the elements in the upper triangle of Saaty's matrix $S$ have to be nondecreasing from left to right in the rows and from the bottom up in the columns. This property was used by the experts to continuously check the weak consistency while entering the data into Saaty's matrix of preference intensities for categories of works of art.

Analogically, we could define weak consistency using elements that are lower than 1 by minimum. This is summarized in the following proposition.

Proposition 1: Let $S=\left\{s_{i j}\right\}_{i, j=1}^{n}$ be Saaty's matrix of preference intensities. Then, $S$ is weakly consistent if and only if the following holds for all $i, j, k \in$ $\{1,2, \ldots, n\}$ :

$$
\begin{gathered}
s_{i j}<1 \wedge s_{j k}<1 \quad \Longrightarrow \quad s_{i k} \leq \min \left\{s_{i j}, s_{j k}\right\} \\
\left(s_{i j}=1 \wedge s_{j k} \leq 1\right) \vee\left(s_{i j} \leq 1 \wedge s_{j k}=1\right) \quad \Longrightarrow \quad s_{i k}=\min \left\{s_{i j}, s_{j k}\right\} .
\end{gathered}
$$

\section{Proof:}

1. First we prove that weak consistency implies conditions (12) and (13):

(a) Let $s_{i j}<1$ and $s_{j k}<1$, then from the reciprocity of $S$ we get $s_{j i}>1$ and $s_{k j}>1$. The weak consistency implies that $s_{k i} \geq \max \left\{s_{j i}, s_{k j}\right\}$, i.e. $s_{i k} \leq \frac{1}{\max \left\{s_{k j}, s_{j i}\right\}}$. Hence, $s_{i k} \leq \frac{1}{s_{k j}}=s_{j k}$ and $s_{i k} \leq \frac{1}{s_{j i}}=s_{i j}$, in other words $s_{i k} \leq \min \left\{s_{j k}, s_{i j}\right\}$.

(b) Let $s_{i j}=1$ and $s_{j k} \leq 1$. Then, $s_{j i}=1$ and $s_{k j} \geq 1$, weak consistency implies that $s_{k i}=s_{k j}$, from reciprocity $s_{i k}=s_{j k}=\min \left\{s_{i j}, s_{j k}\right\}$.

(c) Let $s_{i j} \leq 1$ and $s_{j k}=1$. Then, $s_{j i} \geq 1$ and $s_{k j}=1$, weak consistency implies $s_{k i}=s_{j i}$, from reciprocity $s_{i k}=s_{i j}=\min \left\{s_{i j}, s_{j k}\right\}$.

2. Now let us suppose that $S$ fulfills (12) and (13). We will prove that such matrix $S$ is weakly consistent:

(a) Let $s_{j i}>1$ and $s_{k j}>1$. Reciprocity implies $s_{i j}<1$ a $s_{j k}<1$. From (12) we get $s_{i k} \leq \min \left\{s_{i j}, s_{j k}\right\}$. Then, $s_{i k} \leq s_{i j}$ and $s_{i k} \leq s_{j k}$. From reciprocity we get $s_{k i} \geq s_{j i}$ and $s_{k i} \geq s_{k j}$, i.e. $s_{k i} \geq \max \left\{s_{k j}, s_{j i}\right\}$.

(b) Let $s_{j i}=1$ and $s_{k j} \geq 1$. Reciprocity implies $s_{i j}=1$ and $s_{j k} \leq 1$. From (13) we get $s_{i k}=s_{j k}=\min \left\{s_{i j}, s_{j k}\right\}$. Thus, from reciprocity $s_{k i}=s_{k j}=\max \left\{s_{k j}, s_{j i}\right\}$.

(c) Let $s_{j i} \geq 1$ and $s_{k j}=1$. Reciprocity implies $s_{i j} \leq 1$ and $s_{j k}=1$. From (13) we get $s_{i k}=s_{i j}=\min \left\{s_{i j}, s_{j k}\right\}$. Thus, from reciprocity $s_{k i}=s_{j i}=\max \left\{s_{k j}, s_{j i}\right\}$.

Relations between elements greater than 1 and lower than 1 can be described by propositions 2 and 3 .

Proposition 2: Let $S=\left\{s_{i j}\right\}_{i, j=1}^{n}$ be a weakly consistent Saaty's matrix of preference intensities. If for $i, j, k \in\{1,2, \ldots, n\}$ it holds that $s_{i j}>1$ and $s_{j k}<1$, then the following holds for $s_{i k}$ :

$$
1<s_{i k} \leq s_{i j}, \text { if } s_{i j}>\frac{1}{s_{j k}}=s_{k j} ;
$$


Proof:

$$
\begin{gathered}
1>s_{i k} \geq s_{j k}, \text { if } s_{i j}<s_{k j} ; \\
s_{j i} \leq s_{i k} \leq s_{i j}, \text { if } s_{i j}=s_{k j} .
\end{gathered}
$$

Considering the relationship between $s_{i j}$ and $s_{k j}$ we need to deal with the following 3 situations separately:

1. Let us consider $s_{i j}>s_{k j}$.

(a) Let us suppose that $s_{i k}<1$. Reciprocity then implies $s_{k i}>1$. From weak consistency we get $\left(s_{k i}>1 \wedge s_{i j}>1\right) \Longrightarrow\left(s_{k j} \geq \max \left\{s_{i j}, s_{k i}\right\}\right)$, which is a contradiction to the assumption that $s_{i j}>s_{k j}$.

(b) Let us suppose that $s_{i k}=1$. As $s_{k j}>1$, we get from weak consistency that $s_{i j}=\max \left\{s_{k j}, s_{i k}\right\}=s_{k j}$, which is again a contradiction to the assumption that $s_{i j}>s_{k j}$.

(c) Consequently, $s_{i k}>1$ must hold. As $s_{k j}>1$, weak consistency implies that $s_{i j} \geq \max \left\{s_{i k}, s_{k j}\right\}$. Thus, $s_{i j} \geq s_{i k}>1$ holds.

2. Now let $s_{i j}<s_{k j}$.

(a) Let $s_{i k}>1$. As in 1c) we get $s_{i j} \geq s_{k j}$, which is a contradiction to the assumption that $s_{i j}<s_{k j}$.

(b) Let $s_{i k}=1$. As in 1b) we get $s_{i j}=s_{k j}$, which is again a contradiction to the assumption that $s_{i j}<s_{k j}$.

(c) Consequently, $s_{i k}<1$ must hold. Analogically to 1a), we now get $1>s_{i k} \geq s_{j k}$.

3. Let $s_{i j}=s_{k j}$. As $S$ is weakly consistent, one of the following situations may occur:

(a) Let $s_{i k}>1$. Then, as $s_{k j}>1$, we get from the weak consistency $s_{i j} \geq \max \left\{s_{i k}, s_{k j}\right\}$. As $s_{i j}=s_{k j}$, to fulfill the implication (10) it has to hold that $s_{i j} \geq s_{i k}>1$.

(b) Now let $s_{i k}<1$. Then, $s_{k i}>1$ and as $s_{i j}>1$, the weak consistency implies $s_{k j} \geq \max \left\{s_{i j}, s_{k i}\right\}$. As $s_{i j}=s_{k j}$, to fulfill the implication (10) it has to hold that $s_{i j} \geq s_{k i}$, i.e. $s_{j i} \leq s_{i k}<1$.

(c) The last situation we need to check is $s_{i k}=1$. As $s_{k j}>1$, the weak consistency implies that $s_{i j}=s_{k j}$. As this equation holds, a situation when $s_{i k}=1$ can occur.

When we put together $3 \mathrm{a})-3 \mathrm{c}$ ), we get $s_{j i} \leq s_{i k} \leq s_{i j}$.

Proposition 3: Let $S=\left\{s_{i j}\right\}_{i, j=1}^{n}$ be a weakly consistent Saaty's matrix of preference intensities. If for $i, j \in\{1,2, \ldots, n\}$ it holds that $s_{i j}<1$ and $s_{j k}>1$, then the following holds for $s_{i k}$ :

$$
1<s_{j k} \leq s_{i k}, \text { if } s_{j k}>\frac{1}{s_{i j}}=s_{j i} ;
$$




$$
\begin{gathered}
s_{i j} \leq s_{i k}<1, \text { if } s_{j k}<s_{j i} \\
s_{k j} \leq s_{i k} \leq s_{j k}, \text { if } s_{j k}=s_{j i} .
\end{gathered}
$$

Proof:

The proof is analogical to the proof of Proposition 2 - to obtain (17), (18) and (19), we again distinguish among three cases: $s_{j k}>s_{j i}, s_{j k}<s_{j i}$ and $s_{j k}=s_{j i}$ and for each of them we investigate $s_{i k}>1, s_{i k}<1$ and $s_{i k}=1$.

The concept of weak consistency (10), (11) represents a weakening of the concept of consistency (2). This is summarized in the following proposition.

Proposition 4: Let a Saaty's matrix of preference intensities $S=\left\{s_{i j}\right\}_{i, j=1}^{n}$ be consistent, i.e. $s_{i k}=s_{i j} \cdot s_{j k}$ for all $i, j, k=1,2, \ldots, n$. Then, $S$ is also weakly consistent.

\section{Proof:}

Let $S$ be consistent (i.e. consistency condition (2) is fulfilled). Then, $s_{i j}>1$ and $s_{j k}>1$ imply $s_{i k}=s_{i j} \cdot s_{j k}>\max \left\{s_{i j}, s_{j k}\right\}$, which means that the first condition of weak consistency (10) is fulfilled. Next, if $s_{i j}=1$, then, $s_{i k}=s_{j k}=\max \left\{s_{i j}, s_{j k}\right\}$ and if $s_{j k}=1$, then $s_{i k}=s_{i j}=\max \left\{s_{i j}, s_{j k}\right\}$. The second condition of weak consistency (11) is also fulfilled.

The implication in the Proposition 4 holds only for the consistency defined by (2). On the other hand, it is naturally not true that a matrix that is deemed "consistent enough" according to some of the criteria found in literature has to necessarily fulfill the weak consistency conditions. For example, according to $C R(S)$ even such matrix $S$ may be considered consistent enough, where the decision maker did not manage to keep the preference ordering of the alternatives - at some place he prefers alternative $C$ to alternative $D$ and at the same time he inputs information that $D$ is preferred to $C$. This situation will be illustrated by the following numerical example.

\subsection{Numerical example}

Let us consider the following Saaty's matrix.

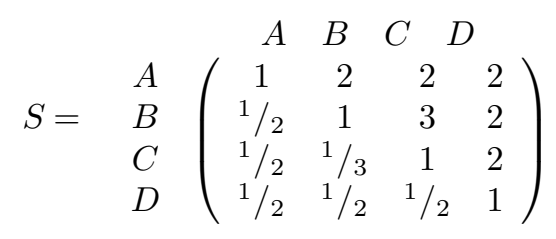

Its maximum eigenvalue is $\lambda_{\max }=4.2153$. The inconsistency index $C I=\frac{4.2153-4}{4-1}=0.0718$ and the inconsistency ratio is $C R(S)=\frac{0.0718}{0.89}=0.0807$. If we use Saaty's weakening of the consistency condition, this matrix will be considered consistent enough as $C R(S)<0.1$. 
From the second row of the matrix $S$ it follows that $B$ is preferred to $C$ and $D$. As the intensity of preference of $B$ to $C$ is larger than $B$ to $D$, we reasonably conclude that $D$ is preferred to $C$. While according to the third row $C$ is preferred to $D$. The preference ordering of the alternatives is clearly violated and still the matrix is considered consistent enough if we use Saaty's inconsistency ratio and the threshold 0.1. We can easily see that $S$ is not weakly consistent: for $s_{23}=3$ and $s_{34}=2$ we would need $s_{24} \geq \max \left\{s_{23}, s_{34}\right\}=3$. However, $s_{24}=2$ which violates condition (10) of the weak consistency.

\section{Determining Scores of the Categories}

The weak consistency of Saaty's matrix can be easily checked during the process of entering data into the matrix. In our case, the experts have decided to additionally (after having completed Saaty's matrix) re-divide the classes of indifferent categories that resulted from the Pairwise Comparison Method. The experts defined the intensities of preferences between pairs of previously indifferent categories and then compared the new categories with the others so that Saaty's matrix remained weakly consistent. Fig. 2 illustrates final Saaty's matrix after re-dividing the pairs of indifferent categories.

If $S$ is close to the ideally consistent matrix, the scores of 27 categories, representing their relative importance, can be calculated by Saaty's method as components of the eigenvector corresponding to the largest eigenvalue of Saaty's matrix $S$.

We can obtain the scores of artistic categories from $S$ also in a different way. The columns of $S$ can be interpreted as repeated measurements of the relative importances of the 27 categories. These measurements are performed by the team of experts who compare all the categories with the first one, then the second one, and so on until the $27^{t h}$ one. From the point of view of mathematical statistics, these are compositional data, i.e. data bearing only relative information (see [1]). Information contained in these data can be expressed by estimating their mean value. A proper estimator of the mean value of this kind of data is a vector whose components are geometric means of the corresponding components of vectors representing single measurements. The relative scores of all 27 categories can be also obtained by computing geometric means of the rows of Saaty's matrix (this calculation method is known as the Logarithmic Least Squares Method, see [6]). If the experts satisfy the condition of weak consistency of the matrix of preference intensities throughout the input process, we can expect the individual measurements and the estimate of the mean value of the compositions to be better.

Fig. 3 compares the scores determined by Saaty's matrix eigenvector method with those determined as geometric means of the rows. The scores are normalized so that the maximum is 305 (analogy to $R \& D$ outcomes evaluation). It is easy to see that the differences between the results of these two methods are not large. Saaty's matrix eigenvector method was used in testing the model on the first real dataset, gathered by Czech art colleges and faculties for the years 2008 to 2010 . 
Stoklasa et al.: Weak consistency in Saaty's AHP

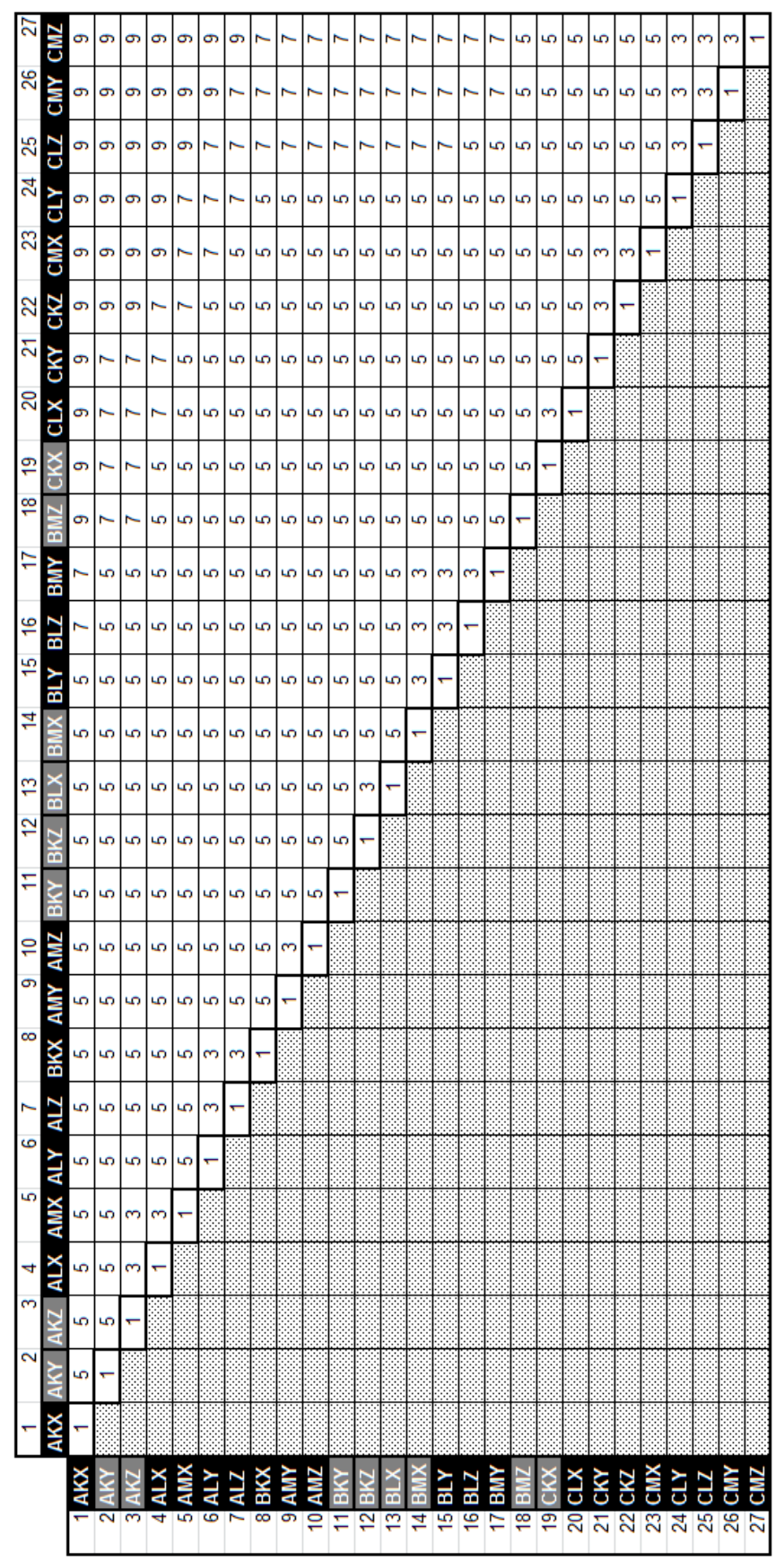

Fig. 2 Saaty's matrix of preference intensities for 27 categories ordered according to their significance. The re-devided categories are highlighted. 


\begin{tabular}{|c|c|c|c|c|c|}
\hline Category & Relevance or significance & Extent & $\begin{array}{l}\text { Institutional } \\
\text { reception }\end{array}$ & $\begin{array}{l}\text { Eigenvector } \\
\text { method }\end{array}$ & $\begin{array}{l}\text { Geom. } \\
\text { means } \\
\text { method }\end{array}$ \\
\hline AKX & crucial significance & large & international & 305 & 305 \\
\hline AKY & crucial significance & large & national & 259 & 254 \\
\hline AKZ & crucial significance & large & regional & 210 & 217 \\
\hline ALX & crucial significance & medium & international & 191 & 194 \\
\hline AMX & crucial significance & limited & international & 174 & 171 \\
\hline ALY & crucial significance & medium & national & 138 & 138 \\
\hline ALZ & crucial significance & medium & regional & 127 & 124 \\
\hline BKX & containing numerous important innovations & large & international & 117 & 112 \\
\hline AMY & crucial significance & limited & national & 97 & 94 \\
\hline AMZ & crucial significance & limited & regional & 90 & 87 \\
\hline BKY & containing numerous important innovations & large & national & 79 & 75 \\
\hline BKZ & containing numerous important innovations & large & regional & 66 & 66 \\
\hline BLX & containing numerous important innovations & medium & international & 62 & 61 \\
\hline BMX & containing numerous important innovations & limited & international & 48 & 50 \\
\hline BLY & containing numerous important innovations & medium & national & 44 & 46 \\
\hline BLZ & containing numerous important innovations & medium & regional & 40 & 41 \\
\hline BMY & containing numerous important innovations & limited & national & 37 & 38 \\
\hline BMZ & containing numerous important innovations & limited & regional & 31 & 30 \\
\hline CKX & pushing forward modern trends & large & international & 26 & 26 \\
\hline CLX & pushing forward modern trends & medium & international & 24 & 24 \\
\hline CKY & pushing forward modern trends & large & national & 19 & 20 \\
\hline CKZ & pushing forward modern trends & large & regional & 17 & 18 \\
\hline CMX & pushing forward modern trends & limited & international & 16 & 16 \\
\hline CLY & pushing forward modern trends & medium & national & 12 & 13 \\
\hline CLZ & pushing forward modern trends & medium & regional & 10 & 11 \\
\hline CMY & pushing forward modern trends & limited & national & 9 & 9 \\
\hline CMZ & pushing forward modern trends & limited & regional & 8 & 9 \\
\hline
\end{tabular}

Fig. 3 Comparison of the results of eigenvector method and the logarithmic least squares method (Geom. means method).

\section{Conclusion}

The paper describes a multiple criteria evaluation model for the works of art resulting from the creative activities of Czech art colleges and faculties. The evaluation model is an integral part of the Registry of Artistic Results (RUV), where information concerning these works of art is stored. The results of this evaluation model have been used as a basis for allocating a part of the state-budget subsidy among art colleges in the Czech Republic since 2012.

For the purpose of determining scores for 27 categories of works of art a two-step procedure is proposed. It was developed in an effort to achieve the best possible conversion of preferences of the expert team into scores for different categories of artistic production. It is based on Saaty's method. Due to the large number of compared objects, our effort was focused on the consistency of Saaty's matrix. Various criteria of sufficient consistency of Saaty's matrix published in the literature were studied and consequently a new notion of weak consistency of Saaty's matrix has been introduced in this paper. For objects descending ordered in accordance with their importance (obtained e.g. by the Pairwise Comparison Method) the weak consistency is easy to check even during the process of entering data into Saaty's matrix of preference intensities. It also constitutes a natural requirement 
for the consistency of information provided by experts concerning their preferences on a set of objects.

The paper shows on a practical application how much effort is needed to obtain information as consistent as possible from a group of experts in a field far away from mathematics (in this case arts).

\section{Acknowledgement}

This research is conducted with the support of the Centralized Developmental Project C41 entitled Evaluating Creative Work Outcomes Pilot Project and financed by the Czech Ministry of Education.

\section{References}

[1] Aitchison J.: The Statistical Analysis of Compositional Data. Monographs on Statistics and Applied Probability. Chapman \& Hall Ltd., London, 1986.

[2] Alonso J. A., Lamata M. T.: Consistency in the Analytic Hierarchy Process: A New Approach. International Journal of Uncertainty, Fuzziness and Knowledge-Based Systems, 14, 4, 2006, pp. 445-459.

[3] Herrera-Viedma E., Herrera F., Chiclana F., Luque M.: Some issues on consistency of fuzzy preference relations. European Journal of Operational Research, 154, 2004, pp. 98-109.

[4] Ji P., Jiang R.: Scale Transitivity in the AHP. The Journal of the Operational Research Society, 54, 8, 2003, pp. 896-905.

[5] Lamata M. T., Pelaez J. I.: A Method for Improving the Consistency Judgements. International Journal of Uncertainty, Fuzziness and Knowledge-Based Systems, 10, 6, 2002, pp. 677-686.

[6] Ramík J.: Analytický hierarchický process (AHP) a jeho využití v malém a středním podnikání. Slezská univerzita v Opavě, Karviná, 2000.

[7] Saaty T. L.: Relative Measurement and its Generalization in Decision Making, Why Pairwise Comparisons are Central in Mathematics for the Measurement of Intangible Factors - The Analytic Hierarchy/Network Process. RACSAM, 102, 2, 2008, pp. 251-318.

[8] Saaty T. L.: The Fundamentals of Decision Making and Priority Theory with the Analytic Hierarchy Process. Vol. VI of the AHP Series, RWS Publ., 2000.

[9] Saaty T. L.: The Brain: Unraveling the Mystery of How it Works, The Neural Network Process. RWS Publ., 1999.

[10] Saaty T. L.: A Scaling Method for Priorities in Hierarchical Structures. Journal of Mathematical Psychology, 15, 1977, pp. 57-68.

[11] Saaty T. L., Vargas L. G.: Decision Making with the Analytic Network Process: Economic, Political, Social and Technological Applications with Benefits, Opportunities, Costs and Risks. Springer, New York, 2006.

[12] Smernica č. 13/2008-R zo 16. októbra 2008 o bibliografickej registrácii a kategorizácii publikačnej činnosti, uměleckej činnosti a ohlasov. Ministerstvo školstva Slovenskej republiky, 2008.

[13] Talašová J.: Fuzzy metody vícekriteriálního hodnocení a rozhodování. Vydavatelství Univerzity Palackého, Olomouc, 2003.

[14] Zelinský M. (ed.): Registr uměleckých výkonů. Akademie múzických umění v Praze, Praha, 2010. 